Mycologia, 96(2), 2004, pp. 249-259.

(C) 2004 by The Mycological Society of America, Lawrence, KS 66044-8897

\title{
Molecular phylogenetic studies on the Diatrypaceae based on rDNA-ITS sequences
}

Francisco Javier Acero

Vicente González

Javier Sánchez-Ballesteros

Víctor Rubio

Departamento de Biotecnología Microbiana, Centro Nacional de Biotecnología (CNB-CSIC), Campus Cantoblanco, Universidad Autónoma de Madrid, Madrid 28049, Spain

Julia Checa

Departamento de Biología Vegetal, Universidad de Alcalá de Henares, Madrid 28871, Spain

Gerald F. Bills

Oscar Salazar

Gonzalo Platas

Fernando Peláez ${ }^{1}$

Centro de Investigación Básica, Merck Sharp and Dohme de España S. A., Josefa Valcárcel 38, Madrid 28027, Spain

Abstract: The order Diatrypales (Ascomycota) contains one single family, the Diatrypaceae. To obtain insight in the phylogenetic relationships within this family, the complete sequences of the ITS region (ITS1, 5.8S rRNA gene and ITS2) of 53 isolates from the five main genera in the family (Diatrype, Diatrypella, Cryptosphaeria, Eutypa and Eutypella) were determined and aligned for phylogenetic reconstruction. Sequence analysis revealed the presence of tandem repeated motifs 11 nucleotides-long, placed in homologous positions along the ITS1 region. Parsimony analysis established the existence of nine monophyletic groups and one branch with a single isolate of Eutypella quaternata. The phylogenetic relationships established by parsimony analysis did not correlate well with classical taxonomic schemes. None of the five genera included in this study was found to be monophyletic. The genera Diatrype, Eutypa and Cryptosphaeria each were divided into two groups. Isolates of Diatrype flavovirens appeared in a clade separated from the one that grouped Diatrype disciformis and the rest of Diatrype species. The Eutypa strains appeared distributed into two clades, one grouping Eutypa lata and related species (Eutypa armeniacae, Eutypa laevata, Eutypa petrakii), and anoth-

Accepted for publication September 22, 2003.

${ }^{1}$ Corresponding author. E-mail: fernando_pelaez@merck.com er with the remaining species of the genus. Eutypella (excluding Eutypella quaternaria) appeared as an unstable monophyletic group, which was lost when the sequence alignment was subjected to neighbor-joining analysis. The genus Diatrypella was not associated with any monophyletic group, suggesting that the multisporate asci character has appeared several times during the evolution of the group. Overall, our study suggests the need to revise many of the concepts usually applied to the classification of members of the family.

Key words: Creosphaeria, Cryptosphaeria, Diatrypaceae, Diatrype, Diatrypella, Eutypa, Eutypella, ITS sequencing, phylogeny, rDNA sequencing, tandem repeat sequences

\section{INTRODUCTION}

The Diatrypaceae is considered the only family of the Diatrypales (Ascomycotina) and currently includes nine accepted genera (Kirk et al 2001). Members of this family are common worldwide, typically occurring on a broad range of dead or declining woody angiosperms. Host specificity is variable within the group, with some species apparently being associated with one plant genus. For instance, Diatrypella betulina (Peck) Sacc. is known only from Betula, whereas others, such as Diatrype flavovirens (Pers. : Fr.) Fr., have a broad host range. Although some members of the Diatrypales are considered parasitic, most are accepted to be saprobic. Species like Eutypella parasitica Davidson \& Lorenz, and especially Eutypa lata (Pers. : Fr.) Tul. \& C. Tul., are known to cause severe diseases on economically important plants (Carter et al 1983).

The selection of morphological traits used to discriminate between genera and species within the Diatrypaceae has varied over time. Earlier systematic arrangements of the family (Fries 1823) were based on stromatal features, and most of the taxa included under the modern concept of the group were recognized primarily as different sections of the genus Sphaeria Haller. The heterogeneity of the group was suggested first by Tulasne and Tulasne (1863) and Currey (1858). An increasing number of diagnostic characters were added subsequently to delimitate taxa within the Diatrypaceae. Features such as mor- 


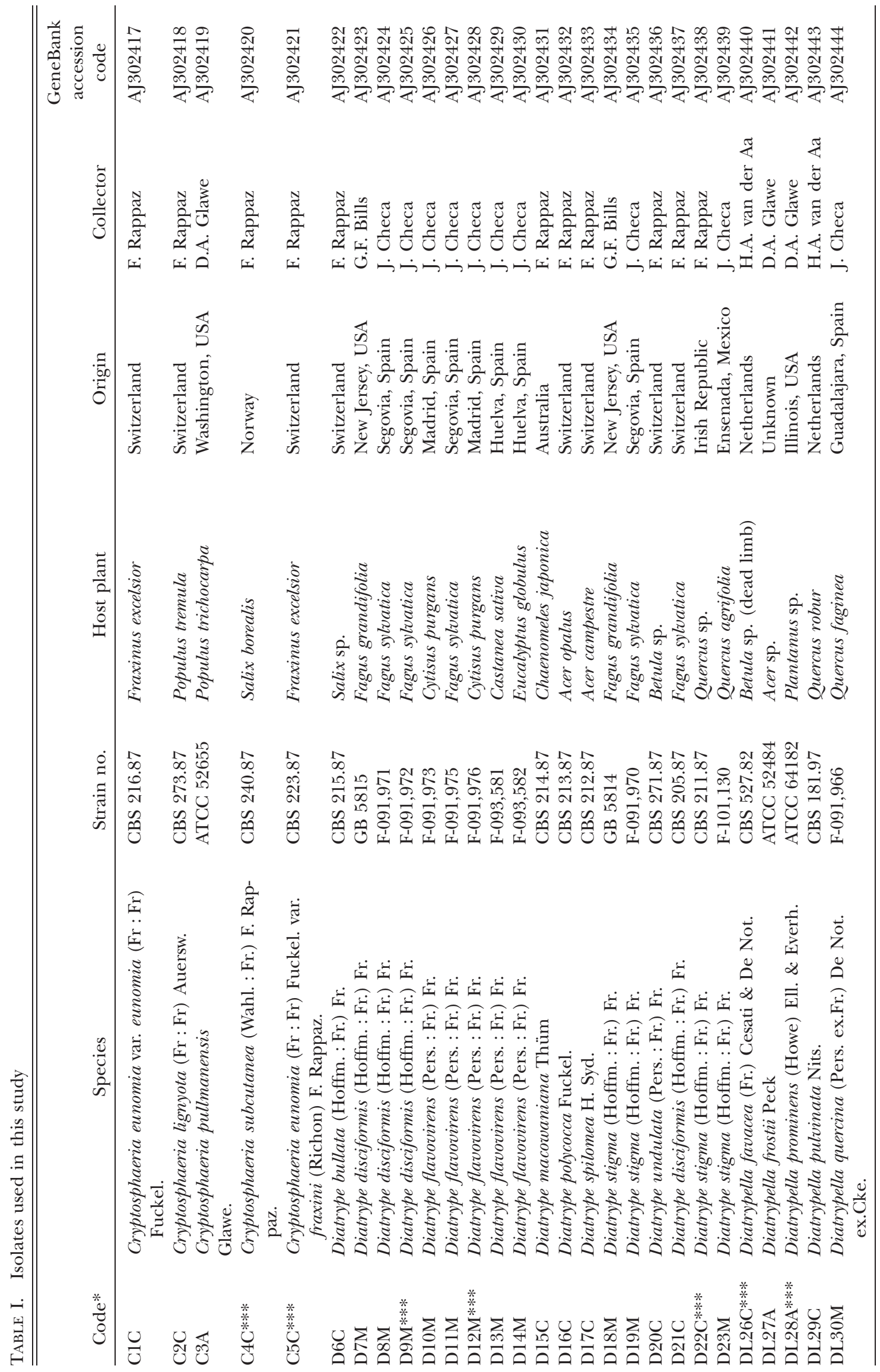


Acero et al: Molecular phylogeny of the DiatrypaceAe

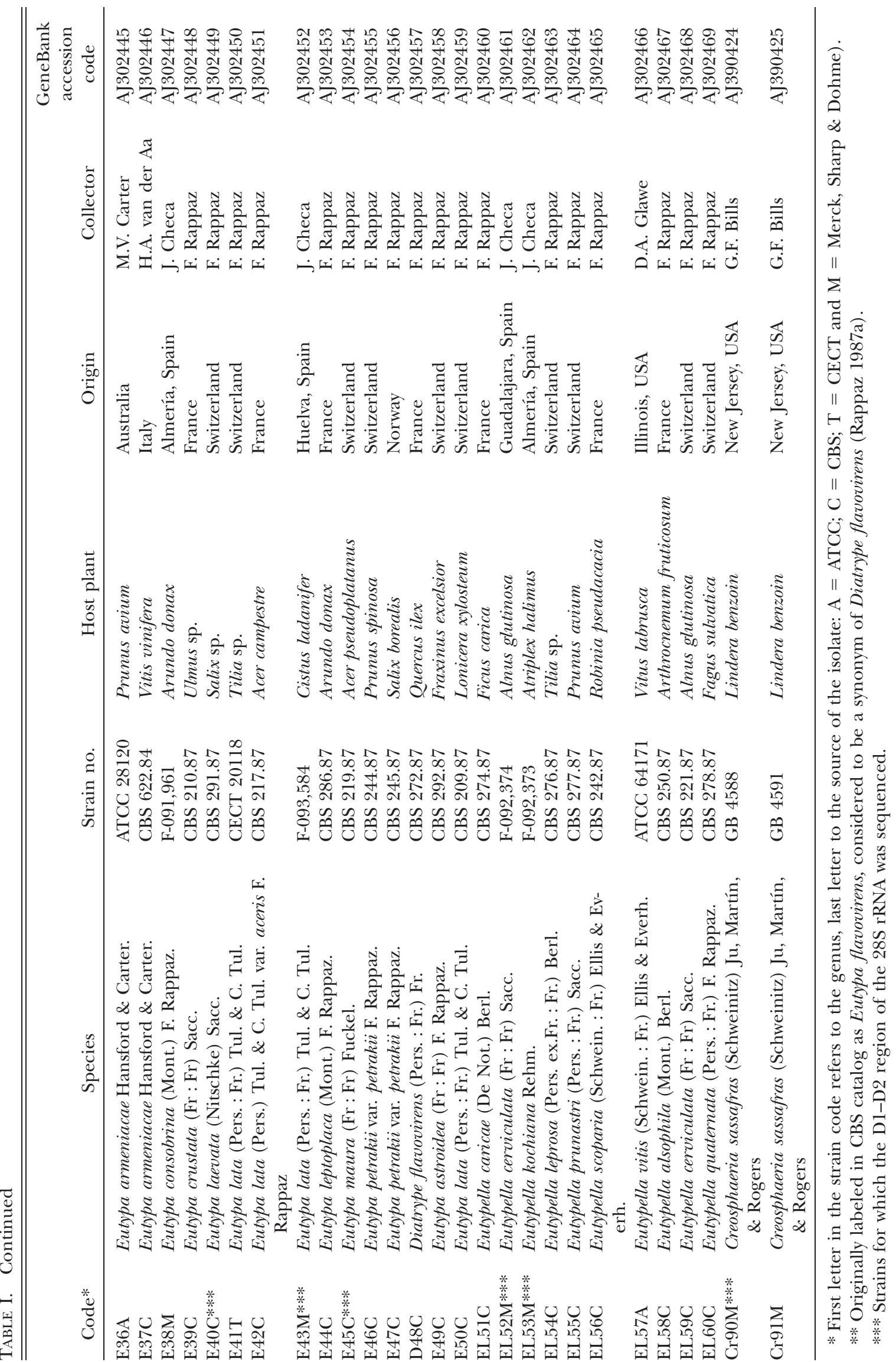


phology and disposition of perithecia (Nitschke 1867), type of anamorph (Winter 1887) or asci and ascospore morphology (Wehmeyer 1926) were incorporated into the descriptions of these organisms. However, stromatal configuration remains an important diagnostic feature to distinguish among the genera within the family (Glawe and Rogers 1984). Thus, valsoid configuration, with perithecia converging at the same point in a poorly developed stroma made up of mixed tissue or fungal hyphae only, usually is ascribed to the genus Eutypella (Nitschke) Sacc. Eutypa Tul. \& C. Tul. and Cryptosphaeria Ces. \& De Not. show eutypoid stromata, characterized by perithecia separately reaching the surface of a flat stroma consisting of mixed tissues from the host and the fungus. These two genera are distinguished by the degree of immersion of the stroma, which is cortical in Cryptosphaeria, whereas in Eutypa it develops in the wood. Finally, in Diatrype Fr. and Diatrypella (Ces. \& De Not.) De Not. the stroma is diatrypoid, consisting of perithecia with short necks that separately reach the surface of a well-developed stroma made up mostly of fungal tissues. These two genera are differentiated by the number of ascospores per ascus; eight in Diatrype and more than eight in Diatrypella.

Unlike other groups of Ascomycetes, anamorph morphology is almost useless when differentiating taxa in the Diatrypaceae, either at the genus or at the species level, because the conidial states in the Diatrypaceae are indistinguishable relatively (Glawe and Rogers 1984, Rappaz 1987a). Three form-genera have been applied to diatrypaceous anamorphs, i.e., Cytosporina Sacc., for fungi with enclosed (pycnidial) conidiomata and filiform conidia; Libertella Desm., for those with unenclosed (acervular) conidiomata and filiform conidia and Naemospora Sacc., for fungi with unenclosed conidiomata and allantoid conidia (Glawe and Rogers 1984). However, there has been a reluctance to assign names to the anamorphs found in culture (e.g., Glawe and Rogers 1984, Rappaz 1987a) because of the unclear limits among those form-genera and because many species produce anamorphs of different types. For instance, Eutypella parasitica produces both pycnidia and acervuli on both natural substrata and agar media (Glawe 1983). Likewise, conidial ontogenesis varies highly in the group and different types of conidiogenesis (e.g., sympodial and percurrent) have been reported in the same strain (Glawe and Rogers 1982a, b). Finally, conidial morphology relatively is indistinct, ranging from allantoid to cylindrical or filiform and from nearly straight to strongly curved.

In this study, the phylogenetic relationships among 53 isolates of the Diatrypaceae were explored based on the comparison of the sequences of the internal- transcribed spacer regions ITS1 and ITS2 (including the 5.8S rRNA gene). The selected isolates represented 35 species from five of the nine accepted genera (Kirk et al 2001) of the Diatrypaceae: Diatrype, Eutypa, Eutypella, Diatrypella and Cryptosphaeria. Echinomyces Rappaz, Fassia Dennis, Leptoperidia Rappaz and Cryptovalsa Ces. and De Not. ex Fuckel are not considered here. This was the first time that this fungal group was subjected to a molecular phylogenetic analysis. The results were compared with morphology-based classification schemes, with the objective of evaluating the phylogenetic significance of characters such as stromatal morphology, ascospore number, anamorph and host. In addition, the phylogenetic relationships between Creosphaeria, a member of the Xylariaceae with a Libertella-like anamorph (Bills and Peláez 1996), and members of the Diatrypaceae were assessed.

\section{MATERIALS AND METHODS}

Fungal isolates and culture conditions.-The isolates used in this work were isolated either by the authors or purchased from the American Type Culture Collection (ATCC, Rockville, Maryland), the Centraalbureau voor Schimmelcultures (CBS, Utrecht, Netherlands) or the Colección Española de Cultivos Tipo (CECT, Valencia, Spain). Care was taken to ensure that the strains obtained from collections had been deposited or identified by well-known specialists in this fungal group to minimize the risk of including misidentified strains in the analysis. The isolates, original substrates, geographical origins and collectors are listed in TABLE I. Isolates were grown on liquid complete media (5 g of each malt extract, yeast extract and glucose $\mathrm{L}^{-1}$ ) in Petri dishes at $26 \mathrm{C}$ for up to $3 \mathrm{wk}$ and maintained on plates at $4 \mathrm{C}$ on potato-dextrose agar (Oxoid, CM139, Basingstoke, Hampshire, U.K.).

DNA sequencing.-All procedures used in this study for DNA purification and ITS amplification have been described previously (Sánchez-Ballesteros et al 2000). Asymmetric PCR amplification was done with a 50:1 molar ratio between the two primers (Gyllenstein and Erlich 1988). The primers used for amplification of the D1 and D2 domains of 28S rRNA gene were LR1 (5'GTAGGAATACCCGCTG AACT3') as concentrated primer and LR4 (based on primer NL4, O'Donnell 1992) for one strand and LR4 as concentrated primer and LR1 for the other strand. The cycling parameters were the same as previously described (Sánchez-Ballesteros et al 2000). PCR products were analyzed by electrophoresis on $1 \%$ agarose gels on TBE buffer (Sambrook et al 1989) and visualized by staining with ethidium bromide. The amplified products were sequenced with an ABI PRISM Dye Terminator Cycle sequencing Kit (Perkin Elmer). All samples were sequenced in both directions, using primer LR3 (5' TGACCATTACGCCAGCATCC $3^{\prime}$ ), when LR1/LR4 were used for amplification, and LR2 (based on NL1 primer; O'Donnell 1992), when LR4/LR1 
were used for amplification. Sequences from each strain were assembled to obtain the sequence of the entire ITS15.8S-ITS2 region and the $5^{\prime}$ region of 28S rRNA gene using the GCG Fragment Assembly System (Program Manual for the Wisconsin Package, version 8). All sequences were deposited in GenBank (TABLE I). Alignments were performed using the CLUSTALW program (Thompson et al 1994) and deposited in TreeBASE (SN734).

Phylogenetic analysis.-Phylogenetic analysis of the aligned sequences was performed by the maximum-parsimony method using the heuristic search algorithm of the Phylogeny Analysis Using Parsimony (PAUP*) program version 4.0 (Swofford 1998). Heuristic search was performed with simple addition of sequences and TBR branch swapping, with MaxTrees set to 100. All characters were unordered and equally weighted, with gaps treated as missing data. The trees were rooted with the ITS sequence of a Neurospora crassa Shear and B.O. Dodge isolate as outgroup. The data were resampled with 1000 bootstrap replicates (Felsenstein 1985). To complement the analysis of branch support we also calculated the decay indexes (Bremer 1994), using the application SEPAL version 1.01 (Salisbury 1999). Neighborjoining analysis also was applied to the same sequence alignment, using the options DNADIST and NEIGHBOR from PHYLIP 3.5c package (Felsenstein 1993). The Jukes and Cantor algorithm was used to estimate the distances between the sequences.

Tandem repeat motifs.-The repeated motifs in the ITS1 region were found using the FINDPATTERNS application from GCG software Wisconsin Package version 10.0.

\section{RESULTS}

The ITS region in the Diatrypaceae were relatively similar in length across the isolates studied, ranging from 503 to $521 \mathrm{bp}$, except five isolates, Diatrypella prominens, Eutypella kochiana and Eutypella quaternata, which had much longer sequences (540, 539 and 539 respectively), and Eutypella leprosa and Eutypella vitis, which had shorter sequences (491 and 493 respectively). Except those five isolates, the length of ITS1 ranged from 188 to $199 \mathrm{bp}$. The size of the ITS2 ranged from 158 to $168 \mathrm{bp}$, always shorter than the ITS1.

The analysis of the ITS1 sequences of all the isolates studied of the Diatrypaceae revealed the presence of DNA motifs repeated in tandem. These were modifications of the 11-nucleotides motif CTACCCTGTAG, found in pure tandem or interspersed in the ITS1 region, in a number ranging from four to seven (data not shown). We detected five repetitions in all the isolates studied, except Diatrypella prominens and Eutypella quaternata, which had seven repetitions and Eutypella leprosa and Eutypella vitis with four. The difference in the number of repetitions would account for the different ITS length of these four isolates.
The different ITS length of E. kochiana is not due to a different number of tandem repeat motifs. This isolate also had five repetitions, and in this case the larger size is explained by insertions of one or more nucleotides along the whole ITS1 region.

The aligned sequences showed a percentage of nucleotide divergence of up to $22.1 \%$ for the complete ITS region, with up to $30.5 \%$ and $31 \%$ divergence in the ITS1 and the ITS2, respectively. The $5.8 \mathrm{~S}$ rRNA gene was conserved among all the strains, except $E$. kochiana, which had a C/T transition at position 120.

One of the 70 most-parsimonious trees derived from the analysis of the whole ITS1-5.8S-ITS2 region is shown in FIG. 1. The complete alignment was 616 bp, with 304 constant characters, 205 parsimony-informative positions and 107 parsimony-uninformative positions. The length of the tree was 1006 steps, with $\mathrm{CI}=0.489, \mathrm{RI}=0.681$, and $\mathrm{RC}=0.333$.

The most basal branch in the tree separated the Creosphaeria isolates from a monophyletic group that included all the Diatrypaceae sensu stricto isolates studied, although the bootstrap support for this main branch was only moderate $(70 \%)$. The next division in the tree left Eutypella quaternata alone in a branch. Above this clade it was possible to distinguish nine groups, seven of them supported by high bootstrap and decay indexes (groups 1-5, 7 and 8). All the groups consisted of members from the same genus, except groups 1, 3 and 8, which contained strains from different genera. The tree did not allow resolving the relationships further among those nine groups, because none of the larger clades observed were supported by bootstrap analysis.

Group 1 was taxonomically the most heterogeneous, although it had one of the highest bootstrap values. It contained two branches, one with Diatrype macowaniana and Eutypella caricae and another with Diatrypella frostii and Diatrypella prominens, and both branches were supported by high bootstrap values. Group 2 clustered all the isolates belonging to Diatrype flavovirens. Group 3 included the Eutypa species analyzed more distant to Eutypa lata (Eutypa leptoplaca, Eutypa consobrina, Eutypa maura, Eutypa crustata and Eutypa astroidea), together with Diatrype polycoc$c a$ and Eutypella prunastri. Group 4 clustered three of the five strains analyzed of genus Cryptosphaeria (Cryptosphaeria lygniota, Cryptosphaeria pullmanensis and Cryptosphaeria subcutanea). Group 5 included two Diatrypella species, Diatrypella pulvinata and Diatrypella favacea. Group 6 contained sequences of the strains of Eutypa lata and related taxa, but this group was not supported by bootstrap analysis. Two Eutypa armeniacae isolates and one Eutypa lata isolate were in this group with identical sequences along the whole region. The next two branches included $E u$ - 


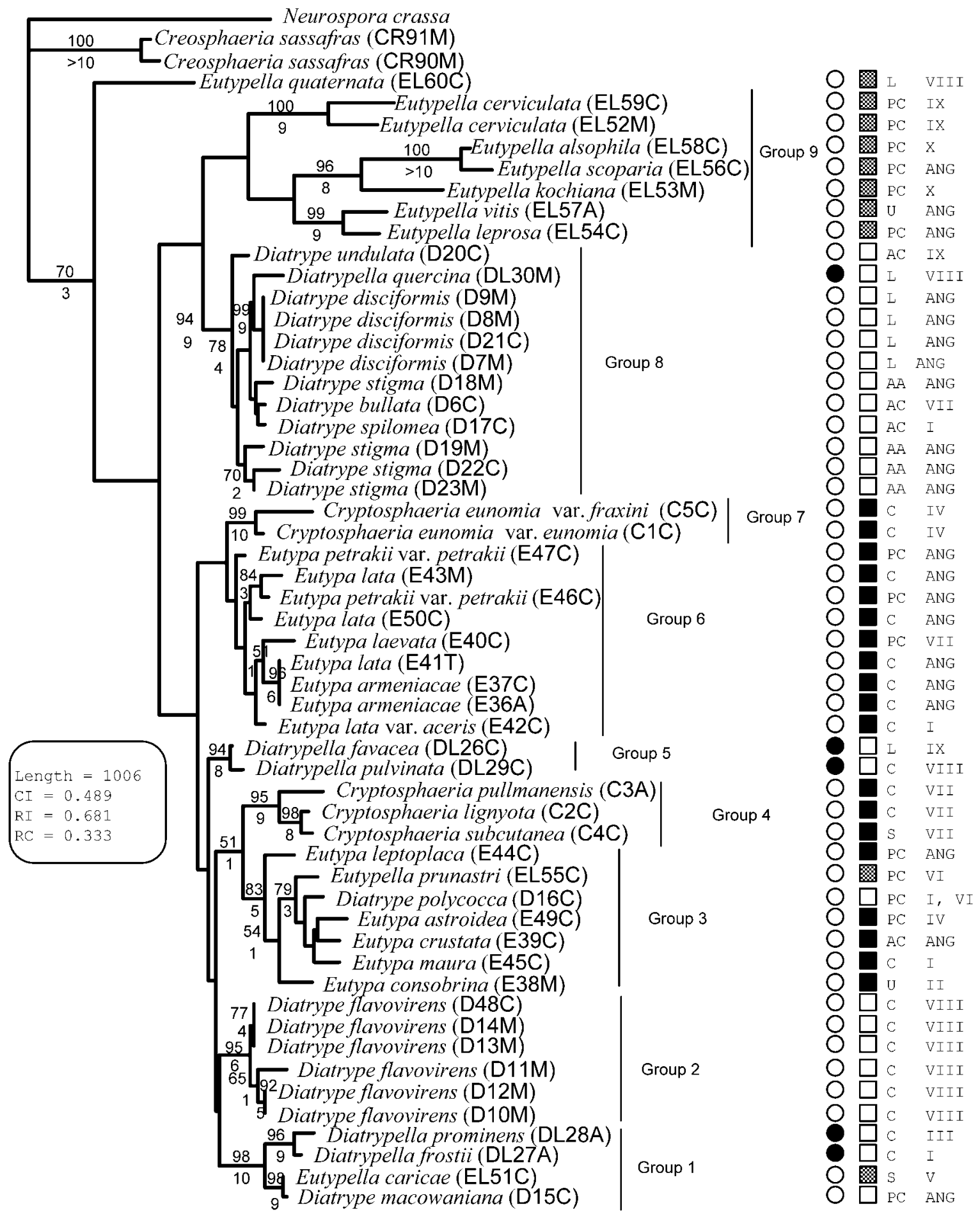

FIG. 1. One of the 70 most equally parsimonious phylogenetic trees generated from the alignment of the ITS1-5.8S-ITS2 region of 55 isolates from the Diatrypaceae. Bootstrap support values are indicated (when more than $50 \%$ ) at the base of the corresponding clade (above the line), together with decay indexes (below the line). Codes used for character mapping. Number of ascospores per ascus: $\bigcirc$ eight, $\bullet$ more than eight. Type of stroma: $\square$ eutypoid, $\square$ diatrypoid, $\otimes$ valsoid. Type of anamorph: C, Cytosporina; L, Libertella; AA, conidiomata acervular, conidia allantoid (sensu Rappaz 1987a); AC, conidiomata acervular, conidia straight to moderately curved (sensu Rappaz 1987a); PC, conidiomata pycnidial, conidia straight to moderately curved (sensu Rappaz 1987a); S, sterile in cultures, no anamorph described (Rappaz 1987a); U, anamorph unknown, species not studied in Rappaz (1987a). Host: ANG Angiosperms (broad host range), I Aceraceae, II Poaceae, III Platanaceae, IV Oleaceae, V Moraceae, VI Rosaceae, VII Salicaceae, VIII Fagaceae, IX Betulaceae, X Chenopodiaceae. 
typa laevata and Eutypa lata var. aceris. The clade also included other two Eutypa lata and two Eutypa petrakii var. petrakii isolates. Group 7 clustered the remaining two isolates of genus Cryptosphaeria (two varieties of Cryptosphaeria eunomia) not included in Group 4. The most populated clade was Group 8, which clustered 11 isolates of genus Diatrype and Diatrypella quercina. All Diatrype disciformis isolates appeared together in a cluster, with identical ITS sequences. The nearest isolate to the Diatrype disciformis group was Diatrype quercina. A second branch clustered three Diatrype stigma isolates (D23M, D22C and $\mathrm{D} 19 \mathrm{M}$ ) and another branch clustered Diatrype spilomea, Diatrype bullata and another Diatrype stigma strain (D18M). Diatrype undulata appeared in a basal branch as the most external isolate. Most Eutypella species were clustered in Group 9, but genetic distances in this group were much higher than in the other groups. This was not considered a reliable monophyletic group because it was not supported by bootstrap analysis. Three main branches were in this clade. One included two Eutypella cerviculata isolates. A second cluster grouped Eutypella alsophila and Eutypella scoparia, with Eutypella kochiana next to them, alone in a single branch. The last branch grouped Eutypella leprosa and Eutypella vitis.

In summary, parsimony analysis of the whole ITS15.8S-ITS2 region revealed little correlation between the molecular data and the morphological criteria classically used for delimiting genera within the Diatrypaceae because no genus could be shown to be monophyletic. Group 1 contained members of Diatrype, Eutypella and Diatrypella as a well supported monophyletic group just like Group 8, which contained taxa from Diatrype and Diatrypella. The moderately well-supported Group 3 contained species of Eutypa, Diatrype and Eutypella. The Cryptosphaeria species were placed in two separate groups. Also, the Diatrype flavovirens isolates formed a well-supported group, separate from the remaining species of Diatrype. Finally, Eutypella quaternata was excluded from the clade containing the remaining Eutypella species (Group 9), although this group was not supported by bootstrap analysis.

Neighbor-joining analysis of the same alignment resulted in a tree that upheld the topology of the nine main clusters resolved in the parsimony tree except Group 9, whose members were segregated in two groups. The internal topology of the strongly supported branches resolved by parsimony analysis also was maintained (data not shown).

Sequences from the D1-D2 region of the 28S rRNA gene from a subgroup of representative species also were obtained to complement the phylogenetic analysis. The size of this region was $556 \mathrm{bp}$ for the 12 isolates analyzed except Eutypella kochiana, with 561 bp. The alignment of these sequences revealed that this region was conserved highly. Only 33 positions from the alignment were parsimony informative, making impossible any reliable reconstruction of the phylogenetic relationships from these data.

\section{DISCUSSION}

One of the most interesting findings in this work is the detection of tandem-repeat sequence patterns in the ITS1 region. The presence of this type of sequences in fungal genomes is well documented (Andersen and Torsten 1997, Giraud et al 1998, Zézé et al 1999), but its occurrence in the ITS regions has been reported only recently. These motifs previously have been reported for Eutypa lata by DeScenzo et al (1999), although in this work the number of repetitions varied among isolates, whereas in our study the number of motifs was consistent among strains from the same species. These tandem-repeat sequences have been found in the ITS region of many members of the Xylariales but not in other fungal groups (Platas et al 2001). In the Diatrypaceae they were located in the ITS1 region at homologous positions, between positions 55-65 and 111-155. Such repeats are lost easily or incorporated by mechanisms of slipping strand mispairing (Platas et al 2001).

Our molecular data poorly correlated with the morphological criteria used for delimiting genera and species within the Diatrypaceae, suggesting that the current taxonomical schemes in the Diatrypaceae might not reflect the natural relationships and limits of the genera traditionally placed in this group. Parsimony analysis of the ITS sequences seem to support a monophyletic origin for the family, although the bootstrap support is weak.

Genus Eutypa.-Our molecular data suggested that genus Eutypa is polyphyletic. The species analyzed appeared distributed in two separate groups. This is consistent with the heterogeneity of the genus hypothesized by many authors, most likely as a consequence of the low number of diagnostic features exhibited. Although without bootstrap support, each Eutypa clade associated with some Cryptosphaeria species, in agreement with the presumed relationship between the two genera suggested by their similarity in stromatal morphology (Wehmeyer 1975, Glawe and Rogers 1984).

The distribution of taxa within Group 6 in the phylogenetic trees suggests a large sequence variability among the strains belonging to Eutypa lata and related taxa. This taxon could be regarded as a species complex, where delimitation of individuals repre- 
senting Eutypa lata sensu stricto could be difficult, given that this species is reported commonly from a large number of plant hosts and old descriptions of the species often lack enough diagnostic characters. Within this group we found a robust subclade containing one strain of Eutypa lata and two strains of Eutypa armeniacae showing identical sequences. Although this could support the hypothesis that both species are synonyms, as suggested by several authors (Glawe 1992, Rappaz 1987a), two other Eutypa lata isolates are peripheral to this subclade. DeScenzo et al (1999) assessed the genetic diversity in a group of Eutypa lata-like isolates from California using ITS sequencing and AFLP fingerprint and their results supported the separation of the two species. The next two branches in Group 6 included Eutypa lata var. aceris, which differs from Eutypa lata only in cultural features and Eutypa laevata, a taxon considered by Rappaz (1987a) as a possible variant of Eutypa lata with smaller ascospores and habitat restricted to Salix spp. The high homology between one of the Eutypa lata (E43M) and one of the Eutypa petrakii isolates (E46C) is remarkable.

Group 3 contained the Eutypa species less related to the Eutypa lata complex. The inclusion of Diatrype polycocca in this clade is intriguing. Our molecular data suggest that Diatrype polycocca is highly related to species of Eutypa, but Rappaz (1987a) described Diatrype polycocca unambiguously as a member of genus Diatrype, with a diatrypoid stroma. It is interesting to note that this species shows a pycnidial anamorph, similar to the typical Eutypa anamorphs, whereas the species of Diatrype in Group 8 usually produce a Libertella-like anamorph (i.e., with unenclosed conidiomata). Another species in this clade not ascribed to Eutypa is Eutypella prunastri. Tiffany and Gilman (1965) have suggested that Eutypella prunastri should be considered as belonging to genus Eutypa, and our data provide additional support for that suggestion.

Genus Cryptosphaeria.-Our molecular analysis suggests a polyphyletic origin for genus Cryptosphaeria. The five species analyzed appear distributed in two separate clades (groups 4 and 7), each related to one of the two Eutypa clades. For the species studies, there is an apparent correlation between this segregation and their host plant range. Thus, the three species included in Group 4 commonly are recorded from members of the Salicaceae, whereas the two taxa included in Group 7 are typical from the Oleaceae. Within Group 4, Cryptosphaeria subcutanea and Cryptosphaeria lignyota were grouped together. They are closely related species, according to Rappaz (1987a). The two varieties of Cryptosphaeria eunomia included in Group 7 showed enough genetic variability to be distinguished from each other. They have been reported to be macroscopically identical, except that var. fraxini posses a distinctive ascospore septum (Rappaz 1987a). Although they appeared grouped, the nucleotide divergence rate between them was relatively high $(4.8 \%)$, compared with other groups in the study. This would support maintaining these two varieties as distinct taxa.

Genus Diatrype.-Parsimony and neighbor-joining analyses suggests a polyphyletic origin for the genus, or at least that Diatrype flavovirens should be segregated from the rest of the species of the genus analyzed. Thus, the strains studied here grouped in two distinct clades, one of them containing sequences from Diatrype flavovirens strains and the other including the remaining Diatrype spp. studied (except Diatrype polycocca and Diatrype macowaniana). The systematic position of Diatrype flavovirens (Group 2) has been reported to be unclear, because it exhibits morphological characters intermediate between Diatrype and Eutypa. Rappaz (1987a) considered Diatrype flavovirens difficult to delimitate because of the limits between well-developed and poorly developed diatrypoid stromata. Our data suggests that it could be considered a taxon different from both Diatrype and Eutypa, but further molecular analyses involving other genes is required to assign the members of this taxon to an independent genus.

Group 8 contained the remaining species of Diatrype, including Diatrype disciformis, the type species of the genus. The sequence of Diatrypella quercina also was included in this group. This taxon has been considered a Diatrypella because of its multisporate asci, but Wehmeyer (1926) discussed the convenience of including this species in Diatrype. In addition, Ruhland (1900) pointed out that Diatrypella quercina could be considered under the concept of Diatrype because of the strongly developed ectostromata and Croxall (1950) later distinguished it from other Diatrypella species because of its strongly curved ascospores. The molecular data presented here suggest that Diatrypella quercina should be considered a member of Diatrype despite its multisporate asci.

All species included in Group 8 have been reported as related to some degree. In addition, Diatrype disciformis clearly was separated from the remaining Diatrype species. All isolates belonging to this taxon showed identical ITS sequences, despite their different geographic origins, and they were arranged together in a monophyletic group with a high bootstrap index. In contrast, the isolates of Diatrype stigma did not cluster in a monophyletic group, suggesting 
that this might be a species complex as hypothesized by several authors. Thus, Wehmeyer (1926) and Nitschke (1867) found differences in conidial sizes in different collections of Diatrype stigma, suggesting that more than one species were included under this epithet. Likewise, Glawe and Rogers (1984) considered five groups for Diatrype stigma based on ascospore size, conidial size and stromatal features. Rappaz (1987b) considered three taxa for Diatrype stigma: Diatrype stigma sensu stricto, Diatrype decorticata and Diatrype undulata. Our study does not include Diatrype decorticata, but FIG. 1 suggests a clear distinction between Diatrype stigma sensu stricto and Diatrype undulata. On the other hand, Diatrype bullata and Diatrype disciformis also were seen as similar to Diatrype stigma by Wehmeyer (1926) and Rappaz (1987b) because of their similar stromatic development. Nevertheless, our analysis did not resolve the relationships among these species.

Genus Diatrypella.-The five isolates of Diatrypella included in this study were distributed into three different clades with high statistical support, in some cases (groups 1 and 8) together with members of other genera. This would suggest that the multisporate ascus trait might have appeared independently several times during the evolution of the Diatrypaceae. Group 5, containing Diatrypella pulvinata and Diatrypella favacea, the type species of genus Diatrypella, could be the representative group of the genus, provided that any character, other than the number of spores per ascus, were used to define this genus. The small nucleotide divergence between these two isolates $(1.1 \%)$ would suggest that they are related closely or even conspecific. The sequencing of additional genes and isolates from these species would be required to confirm this possibility. As already mentioned, Diatrypella quercina should be considered a member of genus Diatrype. Finally, the two Diatrypella species in Group 1 probably should be considered out of the concept of the genus, according to its relative position in the tree. It is interesting to note that Group 1 includes species from three different genera, with very low percentages of divergence, suggesting close affinities among the four taxa. The similarity between the sequences of Diatrype macowaniana and Eutypella caricae is particularly striking $(0.2 \%)$. Although we cannot rule out the possibility that the nomenclatural heterogeneity in this clade could be due to strain misidentifications, our data suggest at least the need of a taxonomic revision of these species.

Genus Eutypella.-The clade containing most of the species from genus Eutypella (Group 9) appeared as an unstable monophyletic group in the analysis of the entire ITS region, with low bootstrap values (FIG. 1). Moreover, such arrangement was not conserved in the neighbor-joining tree (data not shown). It also is interesting to note that several of the strains analyzed showed large differences in length in the ITS1 region, in some cases with a different distribution of the tandem-repeat sequences. This was the most heterogeneous group at the sequence level, with nucleotide divergence percentages ranging between 3.2 and $22.1 \%$. In fact, the appearance of monophyly could be caused by a "long-branch attraction" phenomenon (Maley and Marshall 1998). This group also included species from other ancient genera (e.g., Quaternaria Tul. and C. Tul, Scoptria Nitschke) lately synonymized under Eutypella to maintain name stability (Rappaz 1987a, 1989). However, some of the relationships among the isolates analyzed are conserved and well supported. Thus, the two strains of Eutypella cerviculata clustered together, Eutypella leprosa and Eutypella vitis also were grouped, and there was a third group with Eutypella alsophila, Eutypella scoparia and Eutypella kochiana. Rappaz (1987a) reported that Eutypella kochiana was close to Eutypella alsophila, although the former had smaller ascospores. However, Eutypella alsophila appeared more related to Eutypella scoparia than to Eutypella kochiana in the phylogenetic trees. Furthermore, both Eutypella scoparia and Eutypella alsophila showed a similar distribution pattern of tandem-repeat motifs (data not shown). Moreover, Eutypella kochiana was the only isolate in this study with a different nucleotide in the 5.8S rRNA gene and with five additional nucleotides in the $5^{\prime}$ region of $28 \mathrm{~S}$ rRNA. To get insight to the implications of such observations, the sequencing of other isolates of Eutypella kochiana would be desirable.

Our analysis suggests that Eutypella quaternata (EL60C) should be considered a member of a different genus. This taxon was described as the type species of the genus Quaternaria (as Quaternaria quaternata) and later proposed to be included in genus Eutypella (Rappaz 1987a, Eriksson 1988). Molecular data support maintaining Quaternaria as an independent genus, as proposed by Gams (1994).

Genus Creosphaeria.-One of the goals of this work was to assess the phylogenetic relationships between Creosphaeria and members of the Diatrypaceae. Creosphaeria is classified within the Xylariaceae, but its Libertella-like anamorph (Bills and Peláez 1996) suggests that it could have affinities with the Diatrypaceae (Rappaz 1987a). A previous study based on ITS sequences revealed that Creosphaeria sassafras was peripheral to other members of the Xylariaceae (Sánchez-Ballesteros et al 2000). However, our phyloge- 
netic reconstruction did not reflect a clear link between this genus and the Diatrypaceae. In the parsimony analysis the two Creosphaeria sassafras, sequences appeared in a basal node, out of the main clade that included all the sequences of the Diatrypaceae. Although based on the number and position of the tandem repeat motifs in the ITS1 region, Creosphaeria sassafras would be closer to the Diatrypaceae than to the Xylariaceae (data not shown), the phylogenetic relevance of this finding is unknown. In any case, and although more sequence-based work would be desirable to clarify the systematic placement and evolutionary affinities of the genus, our results suggest maintaining Creosphaeria out of the Diatrypaceae.

Molecular phylogeny and morphological traits.-Most of the monophyletic clades identified in the phylogenetic trees were homogeneous with respect to the type of stromata of the species clustered within. The exceptions were groups 1 and 3, which contained taxa showing different stromatal types. However, the groups containing taxa with diatrypoid or eutypoid configuration were intermingled along the cladogram. The valsoid type was apparently more homogeneous because all of the Eutypella species (except Eutypella prunastri and Eutypella caricae) were arranged in a group, although without bootstrap support. Likewise, species of Eutypa and Cryptosphaeria, which share a similar type of stromata, were grouped together but also lacking bootstrap support. However, the data presented here are insufficient to draw any conclusions about the evolutionary relationships among the stromatal configurations used to define genera in the Diatrypaceae. Our results suggest a possible polyphyletic origin for these stromatal types; the three main types seem to have appeared several times along the natural history of the group. Likewise, the number of ascospores per ascus is not a character associated with any monophyletic group.

In the phylogenetic trees presented here, we have mapped the anamorphs for the species studied based on two sources. The database ANATELEO (http:// www.cbs.knaw.nl/databases/anateleo.html) provides anamorph epithets for some of these species. These have been incorporated in the trees and referred to as Cytosporina or Libertella. However, the anamorphs for most of the species studied have not been named in the literature. In those cases we have assigned a code defining the type of conidiomata and conidia, as described by Rappaz (1987a). The distribution of species in FIG. 1 does not reveal any apparent overall correlation with their anamorphs. The segregation of Diatrype flavovirens from other Diatrype spp. correlates with its different anamorph Cytosporina-like, with pycnidial conidiomata, compared with the anamorphs with conidiomata unenclosed produced by the members of Group 8. However, this apparent correlation is weakened by the observation that Diatrype flavovirens is known to produce pycnidia on the host but unenclosed conidiomata in agar media (Glawe 1983).

Like morphological characters, host range did not show any apparent correlation with the distribution of species in the phylogenetic trees. Species with broad host range appeared distributed across the cladogram and those with restricted host range were not clustered.

In summary, this work presents a preliminary assessment of the phylogenetic relationships among genera of the family Diatrypaceae by sequencing of the ITS region. Our molecular phylogenetic analysis shows little correlation with the current morphological concepts used for delimiting genera in the family. The current generic divisions within this family might not reflect the natural relationships among different taxa.

In addition to its contribution to the understanding of the systematics of the Diatrypaceae, our work may be a useful tool for the identification of diatrypaceous fungi in culture, which is hampered by the fact that anamorphs are almost indistinguishable and not always produced in culture. PCR primers have been designed recently that are useful in the rapid identification of Eutypa lata in culture (Lecomte et al 2000). Although the design of primers for the identification of other species would require sequencing more isolates from diverse geographic locations, our work provides a foundational database that can be used as a reference to compare sequences of unknown isolates of this important family.

\section{LITERATURE CITED}

Andersen TH, Torsten NT. 1997. A fungal minisatellite. Nature 386:771.

Bills GF, Peláez F. 1996. Endophytic isolates of Creosphaeria sassafras. Mycotaxon 57:471-477.

Bremer K. 1994. Branch support and tree stability. Cladistics 10:295-304.

Carter MV, Bolay A, Rappaz F. 1983. An annotated host list and bibliography of Eutypa armeniacae. Rev Plant Pathol 62:251-258.

Croxall HE. 1950. Studies on british pyrenomycetes III. The British species of the genus Diatrypella Cesati and de Notaris. Trans Br Mycol Soc 33:45-72.

Currey F. 1858. Synopsis of the fructification of the compound Sphaeriae of the Hookeria herbarium. Trans Linn Soc London 22:313-335.

DeScenzo RA, Engel SR, Gomez G, Jackson EL, Munkvold GP, Weller J, Irelan NA. 1999. Genetic analysis of Eu- 
typa strains from California supports the presence of two pathogenic species. Phytopathology 89:884-893.

Eriksson OE, Hawksworth DL. 1988. Notes on ascomycete systematics-N. 552-732. Systema Ascomycetum 7:59101.

Felsenstein J. 1985. Confidence intervals on phylogenies: an approach using the bootstrap. Evolution 39:783-791.

. 1993. PHYLIP manual version 3.5. Seattle: University of Washington.

Fries EM. 1823. Systema mycologicum 3, Index: 1-202. Greifswald.

Gams W. 1994. Report of the committee for fungi: 4. Taxon 43:265-267.

Giraud T, Fortini D, Levis C, Brygoo Y. 1998. The minisatellite MSB1, in the fungus Botrytis cinerea, probably mutates by slippage. Mol Biol Evol 15:1524-1531.

Glawe DA. 1983. Observations on the anamorph of Diatrypella frostii. Mycologia 75:913-915.

- 1992. What's in a name? Eutypa armeniacae vs. Eutypa lata. Phytopathology 82:992.

$\longrightarrow$, Rogers JD. 1982a. Observations on the anamorphs of six species of Diatrype and Diatrypella. Can J Bot 60: 245-251.

- $1982 \mathrm{~b}$. Observations on the anamorphs of six species of Eutypa and Eutypella. Mycotaxon 14:334346.

— -1984 . Diatrypaceae in the Pacific Northwest. Mycotaxon 20:401-460.

Gyllenstein UB, Erlich HA. 1988. Generation of singlestranded DNA by the polymerase chain reaction and its application to direct sequencing of the HLA-DQA locus. Proc Natl Acad Sci USA 85:7652-7656.

Kirk PM, Cannon PF, David JC, Stalpers JA. 2001. Ainsworth and Bisby's Dictionary of the Fungi. 9th ed. CAB International, Wallingford, UK. 655 p.

Lecomte P, Péros J-P, Blancard D, Bastien N, Délye C. 2000. PCR assays that identify the grapevine dieback fungus Eutypa lata. Appl Environ Microbiol 66:447-4480.

Maley LE, Marshall CR. 1998. The coming of age of molecular systematics. Science 279:505-506.

Nitschke T. 1867. Pyrenomycetes Germanici. Die Kernpilze Deutschlands. Breslau, 1:1-160.

O’Donnell K. 1992. Ribosomal DNA internal transcribed spacers are highly divergent in the phytopathogenic ascomycete Fusarium sambucinum (Gibberella pulicaris). Curr Genet 22:213-220.

Platas G, Salazar O, Acero J, Sánchez-Ballesteros J, Rubio V, González V, Portal MA, Borkowski J, Peláez F. 2001.
Presence of a simple Tandem Repeat DNA sequence in the ITS1 region of the Xylariales. Curr Microbiol 43: 43-50.

Rappaz F. 1987a. Taxonomie et nomenclature des Diatrypacées á asques octosporés. Mycologia Helvetica 2:285648.

1987b. Taxonomie et nomenclature de Diatrype stigma, D. decorticata et D. undulata (Diatrypaceae, Ascomycetes). Mycotaxon 30:209-219.

- 1989. Proposal to conserve Eutypella (Nitschke) Sacc. (1875) against Quaternaria Tul and Tul (1863) and Scoptria Nitschke (1867) (Ascomycotina). Taxon 38:665.

Ruhland W. 1900. Untersuchungen zu einer Morphologie der stromabildenden Sphaeriales. Hedwigia 39:1-79.

Salisbury BA. 1999. Strongest evidence: maximum apparent phylogenetic signal as a new cladistic optimality criterion. Cladistics 15:137-149.

Sambrook J, Fritsch EF, Maniatis T. 1989. Molecular cloning: a laboratory manual. 2nd ed. Cold Spring Harbor, New York: Cold Spring Harbor Press. 1592 p.

Sánchez-Ballesteros J, Gonzalez V, Salazar O, Acero J, Portal MA, Julián M, Rubio V, Bills GF, Polishook JD, Platas G, Mochales S, Peláez F. 2000. Phylogenetic study of Hypoxylon and related genera based on ribosomal ITS sequences. Mycologia 92:964-977.

Swofford DL. 1998. PAUP*. Phylogenetic Analysis using Parsimony (* and other methods). Version 4 . Sunderland, MA: Sinauer Associates.

Thompson JD, Higgins DG, Gibson TJ. 1994. CLUSTALW: improving the sensitivity of progressive multiple sequence alignment through sequence weighting, position-specific gap penalties and weight matrix choice. Nucl Acid Res 22:4673-4680.

Tiffany LH, Gilman JC. 1965. Iowa ascomycetes 4: Diatrypaceae. Iowa St J Sci 40:121-161.

Tulasne L-R, Tulasne C. 1863. Selecta Fungorum carpologia 2: 1-319. Paris.

Wehmeyer LE. 1926. A biologic and phylogenetic study of the stromatic sphaeriales. Amer J Bot 13:574-645.

Wehmeyer LE. 1975. The pyrenomycetous fungi. Mycol Mem 6:1-250.

Winter G. 1884-1887. Ascomyceten, in Rabenhorst's Kryptogamen-Flora von Deutschland, Österreich und der Schweiz 1. Abt. 1-2:1-928. Leipzig.

Zézé A, Hosny M, van Tuinen D, Gianinazzi-Pearson V, Dulieu H. 1999. MYCDIRE, a dispersed repetitive DNA element in arbuscular mycorrhizal fungi. Mycol Res 103:572-576. 